\title{
Expression of the neurotrophic tyrosine kinase receptors, ntrk1 and ntrk2a, precedes expression of other ntrk genes in embryonic zebrafish
}

\author{
Katie Hahn ${ }^{1}$, Paul Manuel ${ }^{1}$, Cortney Bouldin ${ }^{\text {Corresp. } 1}$ \\ 1 Department of Biology, Appalachian State University, Boone, NC, United States \\ Corresponding Author: Cortney Bouldin \\ Email address: bouldinc@appstate.edu
}

\section{Background}

The neurotrophic tyrosine kinase receptor (Ntrk) gene family plays a critical role in the survival of somatosensory neurons. Most vertebrates have three Ntrk genes each of which encode a Trk receptor: TrkA, TrkB, or TrkC. The function of the Trk receptors is modulated by the p75 neurotrophin receptors (NTRs). Five $n$ trk genes and one p75 NTR gene ( $n g f r b)$ have been discovered in zebrafish. To date, the expression of these genes in the initial stages of neuron specification have not been investigated.

\section{Purpose}

The present work used whole mount in situ hybridization to analyze expression of the five ntrk genes and ngfrb in zebrafish at a timepoint when the first sensory neurons of the zebrafish body are being established (16.5 hpf). Because expression of multiple genes were not found at this time point, we also checked expression at $24 \mathrm{hpf}$ to ensure the functionality of our six probes.

\section{Results}

At $16.5 \mathrm{hpf}$, we found tissue specific expression of $n t r k 1$ in cranial ganglia, and tissue specific expression of $n$ trk2a in cranial ganglia and in the spinal cord. Other genes analyzed at $16.5 \mathrm{hpf}$ were either diffuse or not detected. At $24 \mathrm{hpf}$, we found expression of both ntrk1 and ntrk2a in the spinal cord as well as in multiple cranial ganglia, and we identified ngfrb expression in cranial ganglia at $24 \mathrm{hpf}$. ntrk2b, ntrk3a and $n t r k 3 b$ were detected in the developing brain at $24 \mathrm{hpf}$.

\section{Conclusion}

These data are the first to demonstrate that $n t r k 1$ and $n t r k 2 a$ are the initial neurotrophic tyrosine kinase receptors expressed in sensory neurons during the development of the zebrafish body, and the first to establish expression patterns of ngfrb during early zebrafish development. Our data indicate coexpression of $n t r k 1, n t r k 2 a$ and $n g f r b$, and we speculate that these overlapping patterns indicate relatedness of function. 


\section{Expression of the neurotrophic tyrosine kinase}

\section{2 receptors, $\boldsymbol{n t r k 1}$ and $\boldsymbol{n t r k} \mathbf{2 a}$, precedes expression of}

\section{3 other ntrk genes in embryonic zebrafish.}

5 Katie Hahn $^{1}$, Paul Manuel ${ }^{1}$ and Cortney M Bouldin ${ }^{1}$

6

$7 \quad{ }^{1}$ Department of Biology, Appalachian State University, Boone, NC, USA

9 Corresponding Author:

10 Cortney M Bouldin ${ }^{1}$

572 Rivers Street, Boone, NC, 28608, USA

Email address: bouldinc@appstate.edu

\section{Abstract}

\section{Background}

17 The neurotrophic tyrosine kinase receptor (Ntrk) gene family plays a critical role in the survival of somatosensory neurons. Most vertebrates have three Ntrk genes each of which encode a Trk receptor: TrkA, TrkB, or TrkC. The function of the Trk receptors is modulated by the p75 neurotrophin receptors (NTRs). Five $n t r k$ genes and one p75 NTR gene ( $n g f r b$ ) have been discovered in zebrafish. To date, the expression of these genes in the initial stages of neuron specification have not been investigated. 


\section{Purpose}

25 The present work used whole mount in situ hybridization to analyze expression of the five $n t r k$

26 genes and $n g f r b$ in zebrafish at a timepoint when the first sensory neurons of the zebrafish body

27 are being established (16.5 hpf). Because expression of multiple genes were not found at this

28 time point, we also checked expression at $24 \mathrm{hpf}$ to ensure the functionality of our six probes.

\section{Results}

At 16.5 hpf, we found tissue specific expression of $n t r k 1$ in cranial ganglia, and tissue specific expression of $n t r k 2 a$ in cranial ganglia and in the spinal cord. Other genes analyzed at $16.5 \mathrm{hpf}$ were either diffuse or not detected. At 24 hpf, we found expression of both ntrkl and ntrk2a in

34 the spinal cord as well as in multiple cranial ganglia, and we identified $n g f r b$ expression in cranial ganglia at 24 hpf. $n t r k 2 b, n t r k 3 a$ and $n t r k 3 b$ were detected in the developing brain at 24 hpf.

\section{Conclusion}

39 These data are the first to demonstrate that $n t r k 1$ and $n t r k 2 a$ are the initial neurotrophic tyrosine

40 kinase receptors expressed in sensory neurons during the development of the zebrafish body, and

41 the first to establish expression patterns of $n g f r b$ during early zebrafish development. Our data

42 indicate co-expression of $n t r k 1, n t r k 2 a$ and $n g f r b$, and we speculate that these overlapping

43 patterns indicate relatedness of function. 


\section{Introduction}

47

48

49

50

51

52

54 55

67 specific subset of sensory neurons (Klein, 1994). 

establishment of crosstalk among the neurotrophin signaling pathways and other signaling pathways; the identification of multiple Trk splice isoforms; and the discovery of p75 NTR, which modulates the function of each Trk (Stucky \& Koltzenburg, 1997; Chao, 2003). p75 NTR is capable of binding with low affinity to each of the neurotrophins that interact with Trk receptors (Schecterson \& Bothwell, 2010). In the presence of TrkA, p75 NTR will increase the

74 affinity of the receptor for NGF (Hempstead et al., 1991; Bibel, Hoppe \& Barde, 1999), which results in a prosurvival effect. In other contexts, uncleaved proneurotrophins can bind to p75 NTR and induce death independently of a Trk receptor (Lee et al., 2001; Teng et al., 2005).

77 Much work has gone into investigating the effects of p75 NTR on survival in cell culture. Interactions between Ntrks and p75 NTR in vivo have been slow to materialize (Bothwell, 2016) but are increasingly being better understood (Tanaka et al., 2016; Chen et al., 2017). Zebrafish is proving to be a useful model system for accelerating discovery of the interplay between Trk receptors and p75 NTR in vivo (Anand \& Mondal, 2020). Using mammalian model systems, the Ntrk and $p 75$ NTR genes have been established as vital for neurogenesis, as their expression is necessary for sensory neuron survival. Unlike mammals, zebrafish have undergone a whole-genome duplication event that happened more than 300 million years ago in the teleost lineage (Amores et al., 1998). As a result, zebrafish have five ntrk genes (ntrk1, ntrk2a, ntrk2b, ntrk3a, and ntrk3b), and so five Trk receptors (TrkA, TrkB1,

87 TrkB2, TrkC1, and TrkC2; Hallböök, 1999). We elected to study the expression of the five $n$ trk genes during zebrafish embryogenesis by looking at two time points in early development when the sensory neurons are found in the zebrafish embryo (Metcalfe \& Westerfield, 1990; Kimmel et al., 1995). Near the time when sensory neurons can first be detected, we found previously 
91 undescribed expression of $n$ trk 1 and $n$ trk $2 a$. Later in embryogenesis, when sensory motor

92 reflexes are beginning, we found expression of $n$ trkl, $n$ trk $2 a$ and the p75 NTR homolog, $n g f r b$,

93 in cranial ganglia and the spinal cord. We also confirmed that $n$ trk $2 b, n$ trk $3 a$ and $n$ trk $3 b$ were

94 expressed in the developing brain.

95

\section{Materials \& Methods}

97

$98 \quad$ Fish care

Fish were kept between $26^{\circ} \mathrm{C}-28^{\circ} \mathrm{C}$ in a 14 hour light $/ 10$ hour dark cycle and were cared

100 for according to The Zebrafish Book: A guide for the laboratory use of zebrafish (Danio rerio)

101 (Westerfield, 2000). Water temperature was kept between $26^{\circ} \mathrm{C}$ and $28^{\circ} \mathrm{C}$, conductivity was

102 maintained between 450-650 microSiemens and $\mathrm{pH}$ was kept above 6.8. Fish were fed dry food 103 daily at 9 am and live brine shrimp in the afternoon. All zebrafish use was approved by the 104 Appalachian State University Institutional Animal Care and Use Committee (Protocols 17-13 105 and 17-15).

106

\section{Embryo collection}

Male and female adult zebrafish were separated in breeding tanks in a 2:3 ratio after the afternoon feeding. Timed matings were used to collect embryos. Fertilized, live embryos were

110 then moved to embryo media $\left(15 \mathrm{mM} \mathrm{NaCl}, 0.8 \mathrm{mM} \mathrm{KCl}, 1.3 \mathrm{mM} \mathrm{CaCl} \bullet 2 \mathrm{H}_{2} \mathrm{O}, 0.1 \mathrm{mM}\right.$ $\mathrm{KH}_{2} \mathrm{PO}_{4}, 0.05 \mathrm{mM} \mathrm{Na}_{2} \mathrm{HPO}_{4}, 2 \mathrm{mM} \mathrm{MgSO}_{4} \bullet 7 \mathrm{H}_{2} \mathrm{O}, 0.07 \mathrm{mM} \mathrm{NaHCO}_{3}$ ) and moved to a $28^{\circ} \mathrm{C}$ 112 incubator. 


\section{Embryo staging and fixation}

115 Embryos were staged according to Stages of embryonic development of the zebrafish

116 (Kimmel et al., 1995). Embryos at $16.5 \mathrm{hpf}$ (the 15 somite stage) were distinguished by the

117 number of somites present, as well as by yolk extension length. By Prim-5 stage or $24 \mathrm{hpf}$,

118 embryos have about 30 somites, faint melanin pigment, and a distinct angle between the head

119 and trunk. Embryos were fixed at the appropriate stage using a 4\% paraformaldehyde solution

120 overnight at $4{ }^{\circ} \mathrm{C}$ before dehydration into methanol and long-term storage at $-20^{\circ} \mathrm{C}$.

121

122

\section{cDNA Isolation and Cloning}

The $n t r k 1$ clone was purchased from Dharmacon (clone ID: 9038008). For all others, cDNA was isolated from zebrafish embryos using Superscript III First-Strand Kit (Invitrogen). $n t r k 2 a, n t r k 2 b, n t r k 3 a$, and $n t r k 3 b$ were cloned from cDNA using Gateway pENTR/D-TOPO

Cloning Kit (Invitrogen). All forward primers contained the sequence CACC for directional cloning with the kit. The sequence of the primers used can be found in Table 1, and a diagram of each gene and probe can be found in Supplementary Figure 1.

\section{Probe synthesis}

Sense and anti-sense RNA probes were generated for each of the genes of interest. RNA probes were synthesized with $10 \mu \mathrm{g}$ of plasmid DNA. Plasmid DNA was linearized using restriction digest. Linearized DNA was extracted using a phenol/chloroform extraction. DNA was resuspended and used in a transcription reaction. The transcription reaction used 10x RNA polymerase buffer, 10x DIG labeling mix (Roche), RNAsin (Invitrogen), and T7 or T3 polymerase (New England Biolabs). Cleanup of the synthesized probe was performed using 
137 precipitation with $\mathrm{LiCl}$. For the non-hydrolyzed probe (ntrk2a), the pellet was resuspended in

138 water and hybridization buffer was added. For hydrolyzed probes (ntrk1, ntrk $2 b$, ntrk3a, ntrk3b

139 and $n g f r b)$, the pellet was resuspended in water and then hydrolyzed with sodium bicarbonate

140 and sodium carbonate. Hydrolysis occurred by adding $5 \mu 10.4 \mathrm{M} \mathrm{NaHCO}_{3}$ and $5 \mu 10.6 \mathrm{M}$

$141 \mathrm{Na}_{2} \mathrm{CO}_{3}$ and incubating at $60^{\circ} \mathrm{C}$ for $\mathrm{t}$ minutes where $\mathrm{t}=($ starting $\mathrm{kb}-$ desired $\mathrm{kb}) / 0.11^{*}($ starting

$142 \mathrm{~kb})($ desired $\mathrm{kb}$ ) with $0.35 \mathrm{~kb}$ used as desired size. Hydrolyzed probes were cleaned up with a

143 NucleoSpin RNA Clean-up kit (Macherey-Nagel).

144

145

In situ hybridization and image collection

146

Single-probe whole-mount in situ hybridizations were performed as described by Thisse

147

and Thisse (2008). For imaging, all embryos were moved stepwise to a solution of $75 \%$ glycerol

148

and 25\% PBS and left overnight. The following day embryos were mounted on a $1.0 \mathrm{~mm}$ thick

149

Gold Seal slide and covered with a Corning 22 x $22 \mathrm{~mm}$ cover slip. All embryos were imaged on

150

an Olympus IX81 at 4x or 10x magnification and processed with Olympus cellSens software.

151

152

153

Results

154

155

156

ntrk1, ntrk2a and $n g f r b$ RNA expression

157

Zebrafish $n t r k$ gene expression was examined at two points in early development, 16.5

hpf and 24 hpf, using whole mount in situ hybridization. At 16.5 hpf, embryos are undergoing

embryonic patterning and both neurogenin and neuroD can be found in neurogenic placodes

159

(Metcalfe \& Westerfield, 1990; Kimmel et al., 1995; Andermann, Ungos \& Raible, 2002). By 24 
160 hpf, embryos have completed embryonic patterning and sensory motor reflexes are beginning

161 (Kimmel et al., 1995), making these useful time points to analyze gene expression during the 162 early stages of neuron specification.

163 In zebrafish, only one ntrk1 gene is present (Martin et al., 1995). Expression of the Ntrk1 164 gene in mice has been implicated in the survival of nociceptors (Smeyne et al., 1994; Crowley et 165 al., 1994), the sensory neurons responsible for detecting potentially harmful stimuli (Purves et 166 al., 2001b). Zebrafish ntrkl expression has not been previously investigated at $16.5 \mathrm{hpf}$, but has been seen in cranial ganglia, trigeminal ganglia, and Rohon-Beard (RB) neurons at $24 \mathrm{hpf}$

168 (Nittoli et al., 2018). Using a full-length hydrolyzed ntrkl probe, we found expression at $16.5 \mathrm{hpf}$ in two sets of cranial ganglia and at $24 \mathrm{hpf}$ in two sets of cranial ganglia as well as expression consistent with the RB neurons of the spinal cord (Figure 1).

Zebrafish have two paralogs of the Ntrk2 gene, $n t r k 2 a$ and $n t r k 2 b$ (Martin et al., 1995).

Because $n t r k 2 a$ is most closely related to Ntrk2 in other species, these genes have been presumed to functional similarly. Previous studies have identified expression of $n t r k 2 a$ in the trigeminal ganglia and RB neurons as early as 24 hpf (Martin et al., 1995; Nittoli et al., 2018). Using whole mount in situ hybridization with a partial length probe, which is designed to hybridize to the extracellular domain and the kinase domain of $n t r k 2 a$, we found $n t r k 2 a$ expression consistent with expression in the trigeminal ganglia and RB neurons at $16.5 \mathrm{hpf}$. At $24 \mathrm{hpf}$, we were also able to confirm expression in cranial ganglia and RB neurons (Figure 2). $n g f r b / p 75 N T R$ is a tumor necrosis factor receptor which binds with low-affinity to each of the neurotrophins (Ngf, Bdnf, NT-4/5 and NT-3; McKay et al., 1996). Using a full-length hydrolyzed probe, no specific $p 75$ NTR expression was detected at 16.5 hpf with diffuse staining 
182 observed throughout the embryo (see Supplemental Figure 2 for a comparison of sense and anti183 sense probes), and by $24 \mathrm{hpf}$, expression was found in two sets of cranial ganglia (Figure 3 ).

184

185

186

187

188

189

190

191

192

193

194

195

196

197

198

199

200

201

202

203

204

\section{ntrk2b, ntrk3a and ntrk3b RNA expression}

The function of $n t r k 2 b$ is unknown, and the expression is distinct from that of $n t r k 2 a$

(Nittoli et al., 2018). Other studies using partial sequence probes suggest that expression is found in the telencephalon, thalamus, hypothalamus, tegmentum, hindbrain, cranial nerves, and RB neurons (Nittoli et al., 2018). Using a full-length hydrolyzed probe, no expression of $n$ trk $2 b$ was seen at $16.5 \mathrm{hpf}$, and expression at $24 \mathrm{hpf}$ was confirmed in the telencephalon (Figure 4). Zebrafish have two paralogs of the Ntrk3 gene, $n t r k 3 a$ and $n t r k 3 b$ (Martin et al., 1995). Because of sequence similarity, $n t r k 3 a$ has been presumed to be functionally similar to Ntrk3 in other species. Previous studies have determined expression of $n t r k 3 a$ in the telencephalon, pineal gland, hypothalamus, cranial ganglia, and RB neurons at 24 hpf (Nittoli et al., 2018). Using a full-length hydrolyzed probe, we found no expression of $n t r k 3 a$ at $16.5 \mathrm{hpf}$, and at $24 \mathrm{hpf}$, we were able to confirm expression in the telencephalon and expression consistent with RB neurons (Figure 5).

The function of $n t r k 3 b$ is unknown, and previous studies have identified some overlap in expression with $n t r k 3 a$; other studies identified expression in the telencephalon, thalamus, tegmentum, and otic vesicle at 24 hpf (Nittoli et al., 2018). Using a hydrolyzed partial length probe, we found no expression of $n t r k 3 b$ at $16.5 \mathrm{hpf}$, confirmed expression in the telencephalon and otic vesicle, and at $24 \mathrm{hpf}$, we identified expression in the midbrain and hindbrain (Figure 6). 


\section{Discussion}

207 When compared to other vertebrates, the teleost lineage has undergone an additional 208 whole-genome duplication event (Taylor et al., 2001), and the single ntrkl gene found in 209 zebrafish may be due to gene loss after the duplication (Heinrich \& Lum, 2000). Ntrk1 in mice

210 has been shown to be important for the survival of nociceptors (Reichardt \& Isabel, 1998). While 211 the expression of zebrafish ntrkl has been characterized at $24 \mathrm{hpf}$, expression at $16.5 \mathrm{hpf}$ was not 212 prior to this study, making the current work valuable for understanding $n t r k 1$ expression at a 213 timepoint when sensory neurons are first identified in the zebrafish body. Gene expression at 214 16.5 hpf was seen in two sets of cranial ganglia, with one larger set more anterior and a smaller 215 set more posterior. Expression at $16.5 \mathrm{hpf}$, which resembles cranial ganglia expression, indicates 216 that $n t r k 1$ expression is present early in neurogenic placode formation. Expression of $n t r k 1$ at 24 217 hpf was seen in two sets of cranial ganglia as well as in the spinal cord in a manner consistent 218 with expression in the RB neurons. Ntrk2 and Bdnf in mice have been shown to be critical for mechanoreceptor function 220 (Klein et al., 1993; Ernfors, Lee \& Jaenisch, 1994; Jones et al., 1994), with ntrk2a being most 221 closely related to Ntrk2 in other species (Nittoli et al., 2018). ntrk2a expression was seen in 222 cranial ganglia, which are in a location consistent with trigeminal ganglia, and in the spinal cord 223 in a pattern which resembles RB neurons at both $16.5 \mathrm{hpf}$ and $24 \mathrm{hpf}$. Expression of $n t r k 2 a$ at $22416.5 \mathrm{hpf}$ is unique as it is the only ntrk gene that we saw expressed in the spinal cord at this time 225 point and early expression suggests an important role for $n t r k 2 a$ in development of the early 226 peripheral nervous system. Expression of $n t r k 2 a$ in the trigeminal overlaps with expression of 227 NT-4/5, a TrkB ligand (Table 2; Nittoli et al., 2018). 
$n t r k 2 b$ expression was detected in a completely different location from $n$ trk $2 a$, suggesting

229

230

231

232

233

234

235

236

237

238

239

240

241

242

243

244

245

246

247

248

249

250

the possibility of functional differences. No expression of $n t r k 2 b$ was detected at $16.5 \mathrm{hpf}$ and

expression at $24 \mathrm{hpf}$ was seen in the telencephalon. Although $n t r k 2 a$ is most closely related to

Ntrk2, the TrkB ligand, BDNF, overlaps with $n t r k 2 b$ and not $n t r k 2 a$ at 24 hpf (Table 2; De Felice

et al., 2014). Interestingly, coexpression of $b d n f$ and $n t r k 2 b$ persists in many tissues into

adulthood (Sahu et al., 2019)

$n t r k 3 a$ is the gene most closely related to Ntrk3 in other species (Nittoli et al., 2018). In

mice, Ntrk3 has been shown to be involved in the survival of proprioceptors (Klein et al., 1994),

but the functionality of $n t r k 3 a$ and $n t r k 3 b$ in zebrafish is unknown. Neither $n t r k 3 a$ nor $n t r k 3 b$

expression was detected at 16.5 hpf. At $24 \mathrm{hpf}$, diffuse staining can be seen in $n t r k 3 a$ embryos

throughout the head, with the most specific staining appearing to be located in the telencephalon.

BDNF expression overlaps with $n t r k 3 a$ expression in the telencephalon (Table 2; De Felice et al., 2014; Nittoli et al., 2018). Expression of $n t r k 3 a$ can also be seen in the spinal cord at $24 \mathrm{hpf}$.

Compared to $n t r k 1$ and $n t r k 2 a, n t r k 3 a$ expression appears more diffuse throughout the spinal

cord instead of distinct puncta. Expression of $n t r k 3 b$ was found in the telencephalon, midbrain,

hindbrain, and otic vesicles at $24 \mathrm{hpf}$. Expression in the telencephalon overlaps with known

expression of the TrkA ligand NGF and the TrkB ligand BDNF, as well as with NGF and NT-3

in the otic vesicle (Table 2; Nittoli et al., 2018).

p75 NTR is a member of the tumor necrosis factor receptor superfamily and is mainly

expressed early in development (Dechant \& Barde, 2002). p75 NTR can interact with all of the ntrk genes and can bind with low affinity to NGF, BDNF, and NT-3, the Trk ligands

(Schecterson \& Bothwell, 2010). p75 NTR complicates the idea that each Trk receptors have a modular model of function, with one ligand binding to a Trk receptor to promote survival of a 
251 specific subset of sensory neuron, because p75 NTR can promote survival of neurons as well as 252 neuronal death (Meeker \& Williams, 2015). While p75 NTR complicates the previously 253 understood model, knowing when and where it is expressed will help with our understanding of 254 the $n$ trk genes, including their functionality during early development. NCBI databases indicate that zebrafish have two p75 NTR homologs, ngfra and $n g f r b$. We elected to study expression of $n g f r b$ because at the time when we conceived this study $n g f r b$ 257 had a reference sequence accession number (NP_001185589.1), while ngfra was merely predicted (XP_003198133.2). No expression of $n g f r b$ was detected at the $16.5 \mathrm{hpf}$ time point, while expression at $24 \mathrm{hpf}$ can be seen in two domains of staining in the cranial ganglia. Interestingly, expression of $n g f r b$ appears similar to the expression pattern determined for $n t r k 1$ 261 (Table 2). TrkA has been shown to cause a prosurvival effect for p75 (Hempstead, 2002). An 262 overlapping expression pattern between $n g f r b$ and $n t r k l$ would make sense as expression of both TrkA and p75 receptors in a similar location would promote neuron survival. While previous studies have used partial gene sequences for making probes, a majority of our data was collected using full-length probes that were hydrolyzed to $300-400 \mathrm{bp}$ fragments (Supplemental Figure 1). We reasoned that this would be an advantageous approach with the Trk family that has been demonstrated to express multiple splice isoforms (Barker et al., 1993; McGregor et al., 1994; Eide et al., 1996) because a hydrolyzed full-length probe should not favor 269 any specific gene region or splice isoform. From this we assert that while we identified fewer regions of gene expression than a previous study (Nittoli et al., 2018), we have very high confidence in the locations of gene expression that we have identified. Some studies have suggested that each of the $n t r k$ genes are functionally distinct in 273 zebrafish due to differential expression patterns (Martin et al., 1995; Nittoli et al., 2018), while 
274 other studies have suggested that $n$ trkl, ntrk2a, and ntrk3a are all functionally similar due to 275 overlapping expression with important nociceptive ion channels (Gau et al., 2017). Our work 276 suggests that there are two groupings of genes with one grouping - ntrkl, ntrk $2 a$ and $n g f r b-$ 277 involved in development of the peripheral nervous system and another grouping - ntrk $2 b$, ntrk $3 a$ 278 and $n$ trk $3 b$ - involved in the development of the central nervous system.

279 While we have determined the locations of gene expression at two critical timepoints 280 during development, these efforts do not allow us to definitively determine functionality. Similar 281 to the experiments in mice used to determine functionality, the zebrafish $n t r k$ genes could be 282 knocked out. For example, $n t r k 2 b$ has been knocked out and shown to produce anxiety through 283 an effect on dopaminergic and serotonergic neuronal populations (Sahu et al., 2019). Our data 284 indicate that $n t r k 1$ and $n$ trk $2 a$, the two $n$ trk genes that we found to be initially expressed in 285 sensory neurons, would be interesting to knockout and analyze for functions. CRISPR is an 286 accessible tool that would allow these knockouts to be done relatively easily and could provide 287 information about the functionality of these genes (Hwang et al., 2013; Tennant, Zerucha \& 288 Bouldin, 2019). From our data, we hypothesize that both $n t r k l$, because of the similarity of the 289 protein to $N$ trkl/TrkA, and $n t r k 2 a$, because of its expression in cranial ganglia, would play a 290 critical role in nociception. Behavioral analysis using zebrafish have been developed (Curtright 291 et al., 2015), which could be performed on crispants to test this hypothesis.

\section{Conclusions}

In our study, we looked at $n$ trk expression in zebrafish at two early time points in development, including $16.5 \mathrm{hpf}$, which is earlier than seen in any other published account of expression in zebrafish (Martin et al., 1995; Martin, Sandell \& Heinrich, 1998; Gau et al., 2017; 
297 Nittoli et al., 2018). In addition, we used hydrolyzed probes to provide a method of determining

298

299

300

301

302

303

304

305

306

307 308

309

310

311

312

313

314

315

316

317

318

319 expression that would not be limited to a specific splice isoform or homologous region. Through this study, we have investigated embryonic expression of the ntrk genes and one $p 75$ NTR (ngfrb) in zebrafish. We have identified expression of all five $n t r k$ genes and $n g f r b$ prior to the larval stages, which strengthens the developmental role of these genes in establishing connections between neurons and target cells, and we have established $n t r k l$ and $n t r k 2 a$ as the genes expressed in the first sensory neurons of developing zebrafish embryos.

\section{Acknowledgements}

The authors would like to thank Andrew Bellemer for helpful discussions and critical reading of the manuscript. Additionally, we would also like to thank Appalachian State University College of Arts and Sciences Vivarium, Monique Eckerd and Scott Rhyne for assistance in caring for our zebrafish colony, and the College of Arts and Sciences William C. and Ruth Ann Dewel Microscopy Facility and Dr. Guichuan Hou, the director of the Dewel Microscopy Facility. This work was supported by the Department of Biology, the College of Arts and Sciences, and the Office of Student Research.

\section{References}

Amores A, Force A, Yan YL, Joly L, Amemiya C, Fritz A, Ho RK, Langeland J, Prince V, Wang YL, Westerfield M, Ekker M, Postlethwait JH. 1998. Zebrafish hox clusters and vertebrate genome evolution. Science 282:1711-1714. 
320

321

322

323

324

325

326

327

328

329

330

331

332

333

334

335

336

337

338

339

340

341

342

343

344

Anand SK, Mondal AC. 2020. Neuroanatomical distribution and functions of brain-derived neurotrophic factor in zebrafish (Danio rerio) brain. Journal of Neuroscience Research 98:754-763. DOI: 10.1002/jnr.24536.

Andermann P, Ungos J, Raible DW. 2002. Neurogenin1 defines zebrafish cranial sensory ganglia precursors. Developmental Biology 251:45-58.

Barker PA, Lomen-Hoerth C, Gensch EM, Meakin SO, Glass DJ, Shooter EM. 1993. Tissuespecific alternative splicing generates two isoforms of the trkA receptor. Journal of Biological Chemistry 268:15150-15157.

Benito-Gutiérrez Ė, Garcia-Fernàndez J, Comella JX. 2006. Origin and evolution of the Trk family of neurotrophic receptors. Molecular and Cellular Neuroscience 31:179-192.

Bibel M, Barde YA. 2000. Neurotrophins: key regulators of cell fate and cell shape in the vertebrate nervous system. Genes \& Development 14:2919-2937.

Bibel M, Hoppe E, Barde YA. 1999. Biochemical and functional interactions between the neurotrophin receptors trk and p75NTR. The EMBO journal 18:616-622.

Bothwell M. 2016. Recent advances in understanding neurotrophin signaling. F1000Research 5.

Chao MV. 2003. Neurotrophins and their receptors: a convergence point for many signalling pathways. Nature Reviews. Neuroscience 4:299-309.

Chen Z, Donnelly CR, Dominguez B, Harada Y, Lin W, Halim AS, Bengoechea TG, Pierchala BA, Lee K-F. 2017. p75 Is Required for the Establishment of Postnatal Sensory Neuron Diversity by Potentiating Ret Signaling. Cell Reports 21:707-720. DOI: 10.1016/j.celrep.2017.09.037.

Crowley C, Spencer SD, Nishimura MC, Chen KS, Pitts-Meek S, Armanini MP, Ling LH, McMahon SB, Shelton DL, Levinson AD. 1994. Mice lacking nerve growth factor display perinatal loss of sensory and sympathetic neurons yet develop basal forebrain cholinergic neurons. Cell 76:1001-1011. 
345 Curtright A, Rosser M, Goh S, Keown B, Wagner E, Sharifi J, Raible DW, Dhaka A. 2015.

346

347

348

349

350

351

352

353

354

355

356

357

358

359

360

361

362

363

364

365

366

367

368

369

Modeling nociception in zebrafish: a way forward for unbiased analgesic discovery. PloS One 10:e0116766. DOI: 10.1371/journal.pone.0116766.

De Felice E, Porreca I, Alleva E, De Girolamo P, Ambrosino C, Ciriaco E, Germanà A, Sordino P. 2014. Localization of BDNF expression in the developing brain of zebrafish. Journal of Anatomy 224:564-574. DOI: 10.1111/joa.12168.

Dechant G, Barde Y-A. 2002. The neurotrophin receptor p75(NTR): novel functions and implications for diseases of the nervous system. Nature Neuroscience 5:1131-1136.

Eide FF, Vining ER, Eide BL, Zang K, Wang X-Y, Reichardt LF. 1996. Naturally Occurring Truncated trkB Receptors Have Dominant Inhibitory Effects on Brain-Derived Neurotrophic Factor Signaling. The Journal of Neuroscience 16:3123.

Ernfors P, Lee KF, Jaenisch R. 1994. Mice lacking brain-derived neurotrophic factor develop with sensory deficits. Nature 368:147-150.

Gau P, Curtright A, Condon L, Raible DW, Dhaka A. 2017. An ancient neurotrophin receptor code; a single Runx/Cbf $\beta$ complex determines somatosensory neuron fate specification in zebrafish. PLoS genetics 13:e1006884.

Hallböök F. 1999. Evolution of the vertebrate neurotrophin and Trk receptor gene families. Current Opinion in Neurobiology 9:616-621.

Heinrich G, Lum T. 2000. Fish neurotrophins and Trk receptors. International Journal of Developmental Neuroscience 18:1-27.

Hempstead BL. 2002. The many faces of p75NTR. Current Opinion in Neurobiology 12:260267.

Hempstead BL, Martin-Zanca D, Kaplan DR, Parada LF, Chao MV. 1991. High-affinity NGF binding requires coexpression of the trk proto-oncogene and the low-affinity NGF receptor. Nature 350:678-683. 
370 Huang EJ, Reichardt LF. 2001. Neurotrophins: Roles in Neuronal Development and Function. $371 \quad$ Annual Review of Neuroscience 24:677-736.

372 Huang EJ, Reichardt LF. 2003. Trk Receptors: Roles in Neuronal Signal Transduction. Annual $373 \quad$ Review of Biochemistry 72:609-642.

374 Hwang WY, Fu Y, Reyon D, Maeder ML, Tsai SQ, Sander JD, Peterson RT, Yeh J-RJ, Joung 375 JK. 2013. Efficient genome editing in zebrafish using a CRISPR-Cas system. Nature $376 \quad$ Biotechnology 31:227-229. DOI: 10.1038/nbt.2501.

377 Jones KR, Fariñas I, Backus C, Reichardt LF. 1994. Targeted disruption of the BDNF gene 378 perturbs brain and sensory neuron development but not motor neuron development. Cell $379 \quad 76: 989-999$.

380 Kimmel CB, Ballard WW, Kimmel SR, Ullmann B, Schilling TF. 1995. Stages of embryonic 381 development of the zebrafish. Developmental Dynamics 203:253-310.

382 Klein R. 1994. Role of neurotrophins in mouse neuronal development. The FASEB Journal 383 8:738-744.

384

Klein R, Silos-Santiago I, Smeyne RJ, Lira SA, Brambilla R, Bryant S, Zhang L, Snider WD, 385 Barbacid M. 1994. Disruption of the neurotrophin-3 receptor gene trkC eliminates la muscle afferents and results in abnormal movements. Nature 368:249-251.

Klein R, Smeyne RJ, Wurst W, Long LK, Auerbach BA, Joyner AL, Barbacid M. 1993. Targeted 388 disruption of the trkB neurotrophin receptor gene results in nervous system lesions and neonatal death. Cell 75:113-122.

Lee R, Kermani P, Teng KK, Hempstead BL. 2001. Regulation of Cell Survival by Secreted Proneurotrophins. Science 294:1945-1948. DOI: 10.1126/science.1065057.

Martin SC, Marazzi G, Sandell JH, Heinrich G. 1995. Five Trk receptors in the zebrafish. Developmental Biology 169:745-758. 
394 Martin SC, Sandell JH, Heinrich G. 1998. Zebrafish TrkC1 and TrkC2 receptors define two

395

396

397

398

399

400

401

402

403

404

405

406

407

408

409

410

411

412

413

414

415

416

417

418

419

different cell populations in the nervous system during the period of axonogenesis. Developmental Biology 195:114-130.

McGregor LM, Baylin SB, Griffin CA, Hawkins AL, Nelkin BD. 1994. Molecular cloning of the cDNA for human TrkC (NTRK3), chromosomal assignment, and evidence for a splice variant. Genomics 22:267-272.

McKay SE, Garner A, Caldero J, Tucker RP, Large T, Oppenheim RW. 1996. The expression of trkB and p75 and the role of BDNF in the developing neuromuscular system of the chick embryo. Development 122:715-724.

Meeker RB, Williams KS. 2015. The p75 neurotrophin receptor: at the crossroad of neural repair and death. Neural Regeneration Research 10:721-725.

Metcalfe WK, Westerfield M. 1990. Primary Motoneurons of the Zebrafish. In: Raymond PA, Easter SS, Innocenti GM eds. Systems Approaches to Developmental Neurobiology. Boston, MA: Springer US, 41-47.

Nittoli V, Sepe RM, Coppola U, D’Agostino Y, De Felice E, Palladino A, Vassalli QA, Locascio A, Ristoratore F, Spagnuolo A, D'Aniello S, Sordino P. 2018. A comprehensive analysis of neurotrophins and neurotrophin tyrosine kinase receptors expression during development of zebrafish. The Journal of Comparative Neurology 526:1057-1072.

Purves D, Augustine GJ, Fitzpatrick D, Katz LC, LaMantia A-S, McNamara JO, Williams SM. 2001a. The Somatic Sensory System. In: Neuroscience. 189-208.

Purves D, Augustine GJ, Fitzpatrick D, Katz LC, LaMantia A-S, McNamara JO, Williams SM. 2001b. Construction of Neural Circuits. In: Neuroscience. 493-517.

Reichardt LF, Isabel F. 1998. Neurotrophic factors and their receptors. Oxford University Press.

Sahu MP, Pazos-Boubeta Y, Pajanoja C, Rozov S, Panula P, Castrén E. 2019. Neurotrophin receptor Ntrk2b function in the maintenance of dopamine and serotonin neurons in zebrafish. Scientific Reports 9:2036. DOI: 10.1038/s41598-019-39347-3. 
420 Schecterson LC, Bothwell M. 2010. Neurotrophin receptors: Old friends with new partners. $421 \quad$ Developmental Neurobiology 70:332-338.

422 Smeyne RJ, Klein R, Schnapp A, Long LK, Bryant S, Lewin A, Lira SA, Barbacid M. 1994. 423 Severe sensory and sympathetic neuropathies in mice carrying a disrupted Trk/NGF $424 \quad$ receptor gene. Nature 368:246-249.

425 Stucky CL, Koltzenburg M. 1997. The Low-Affinity Neurotrophin Receptor p75 Regulates the 426 Function But Not the Selective Survival of Specific Subpopulations of Sensory Neurons. Journal of Neuroscience 17:4398-4405. DOI: 10.1523/JNEUROSCI.17-11-04398.1997.

428 Tanaka K, Kelly CE, Goh KY, Lim KB, Ibáñez CF. 2016. Death Domain Signaling by Disulfide429 Linked Dimers of the p75 Neurotrophin Receptor Mediates Neuronal Death in the CNS. $430 \quad$ Journal of Neuroscience 36:5587-5595.

431 Taylor JS, Van de Peer Y, Braasch I, Meyer A. 2001. Comparative genomics provides evidence 432 for an ancient genome duplication event in fish. Philosophical Transactions of the Royal Society of London. Series B 356:1661-1679.

434 Teng HK, Teng KK, Lee R, Wright S, Tevar S, Almeida RD, Kermani P, Torkin R, Chen Z-Y, 435 Lee FS, Kraemer RT, Nykjaer A, Hempstead BL. 2005. ProBDNF induces neuronal apoptosis via activation of a receptor complex of p75NTR and sortilin. The Journal of Neuroscience 25:5455-5463.

Tennant M, Zerucha T, Bouldin C. 2019. Cost-efficient and effective mutagenesis in zebrafish with CRISPR/Cas9. Eastern Biologist Special Issue 1:64-74.

Thisse C, Thisse B. 2008. High-resolution in situ hybridization to whole-mount zebrafish embryos. Nature Protocols 3:59-69.

442 Westerfield M. 2000. The Zebrafish Book. A Guide for The Laboratory Use of Zebrafish (Danio 443 rerio). 
Table $\mathbf{1}$ (on next page)

ntrk cloning primers 
1 Table 1. ntrk cloning primers

\begin{tabular}{|c|c|c|}
\hline $\begin{array}{l}\text { Gene } \\
\text { name }\end{array}$ & Forward primer & Reverse primer \\
\hline$n t r k 2 a$ & $\frac{\text { CACCATGAGCTTCGGCATG }}{\mathrm{AC}}$ & $\begin{array}{l}\text { TTATCCCAGGATGTCC } \\
\text { AGA }\end{array}$ \\
\hline$n t r k 2 b$ & $\frac{\text { CACCATGACCGCAGGGGTT }}{\mathrm{C}}$ & $\begin{array}{l}\text { TTAGCCCAGGATGTC } \\
\text { CAG }\end{array}$ \\
\hline$n t r k 3 a$ & $\frac{\text { CACCATGGATTTATTCTCCA }}{\text { TCCC }}$ & $\begin{array}{l}\text { CTAGCCCAGGATATC } \\
\text { CAG }\end{array}$ \\
\hline$n t r k 3 b$ & $\frac{\mathrm{CACCGGACTTTAAGTGCCT}}{\mathrm{GC}}$ & $\begin{array}{l}\text { AACATTTAAATCCAA } \\
\text { CAGGTG }\end{array}$ \\
\hline
\end{tabular}

2 


\section{Table 2 (on next page)}

ntrk expression overlaps with known expression patterns of the Trk ligands. 
1 Table 2. Summary of $n$ trk expression patterns and ligands expressed in similar tissues.

\begin{tabular}{|c|c|c|c|}
\hline Gene name & $\begin{array}{l}\text { Corroborated gene expression } \\
\text { locations at } 24 \mathrm{hpf}\end{array}$ & Genes expressed in similar tissues & $\begin{array}{l}\text { Found at } \\
16.5 \mathrm{hpf} \text { ? }\end{array}$ \\
\hline \multirow[t]{2}{*}{ ntrkl } & Cranial ganglia $^{\mathrm{a}, \mathrm{b}}$ & $n t r k 2 a^{\mathrm{a}, \mathrm{b}}, n g f r b^{\mathrm{a}}$ & Yes $^{a}$ \\
\hline & Spinal cord ${ }^{a, b}$ & $n t r k 2 a^{\mathrm{a}, \mathrm{b}}, n t r k 3 a^{\mathrm{a}, \mathrm{b}}, b d n f^{\mathrm{c}}$ & $\mathrm{No}^{\mathrm{a}}$ \\
\hline \multirow[t]{2}{*}{$n t r k 2 a$} & Cranial ganglia ${ }^{a, b}$ & $n t r k 1$ a, b $n g f r b^{\mathrm{a}}$ & Yes $^{\text {a }}$ \\
\hline & Spinal cord ${ }^{\mathrm{a}, \mathrm{b}}$ & $n t r k 1^{\mathrm{a}, \mathrm{b}}, n t r k 3 a^{\mathrm{a}, \mathrm{b}}, b d n f^{\mathrm{c}}$ & Yes $^{a}$ \\
\hline$n t r k 2 b$ & Forebrain $\mathrm{a}, \mathrm{b}$ & $n t r k 3 a^{\mathrm{a}, \mathrm{b}}, n t r k 3 b^{\mathrm{a}, \mathrm{b}}, n g f^{b}, b d n f^{\mathrm{c}}$ & $\mathrm{No}^{\text {a }}$ \\
\hline \multirow[t]{2}{*}{$n t r k 3 a$} & Forebrain $\mathrm{a}, \mathrm{b}$ & $n t r k 2 b^{\mathrm{a}, \mathrm{b}}, n t r k 3 b^{\mathrm{a}, \mathrm{b}}, n g f^{b}, b d n f^{\mathrm{c}}$ & No ${ }^{a}$ \\
\hline & Spinal cord ${ }^{a, b}$ & $n t r k 2 a^{\mathrm{a}, \mathrm{b}}, n t r k 3 a^{\mathrm{a}, \mathrm{b}}, b d n f^{\mathrm{c}}$ & No ${ }^{a}$ \\
\hline \multirow[t]{2}{*}{$n t r k 3 b$} & Forebrain ${ }^{a, b}$ & $n t r k 2 b^{\mathrm{a}, \mathrm{b}}, n t r k 3 b^{\mathrm{a}, \mathrm{b}}, n g f^{b}, b d n f^{\mathrm{c}}$ & No ${ }^{\text {a }}$ \\
\hline & Otic vesicle ${ }^{a, b}$ & $n g f^{b}, n t f 3^{b}, n t f 6 / 7^{b}, b d n f^{c}$ & $\mathrm{No}^{\mathrm{a}}$ \\
\hline
\end{tabular}

2 a. The present work

3 b. Nittoli et al., 2018

4 c. De Felice et al., 2014 


\section{Figure 1}

Early expression of $n t r k 1$ is seen in the head and spinal cord using whole mount in situ hybridization at $16.5 \mathrm{hpf}$ and $24 \mathrm{hpf}$.

$(A \& D)$ Expression of $n t r k 1$ is seen at $16.5 \mathrm{hpf}$ is seen in two sets of cranial ganglia (arrow in $A$ and arrows in $B$ ). (B, C, E \& F) At $24 \mathrm{hpf}$, expression is seen in two sets of cranial ganglia (arrows) as well as in the RB neurons (arrowheads). (A, B, C \& F) Lateral view. (D \& E) Dorsal view. All embryos are oriented with the head to the left. hb, marks the hindbrain, and ye, marks the yolk extension. Data are representative of three rounds of in situ hybridization with least 25 embryos per round.
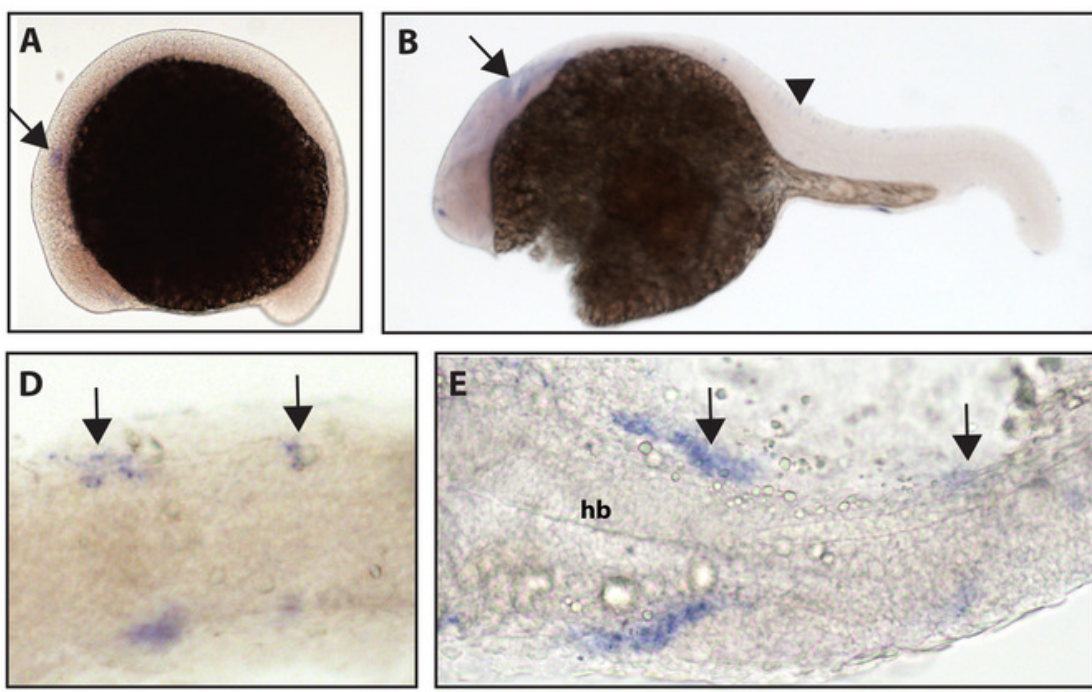
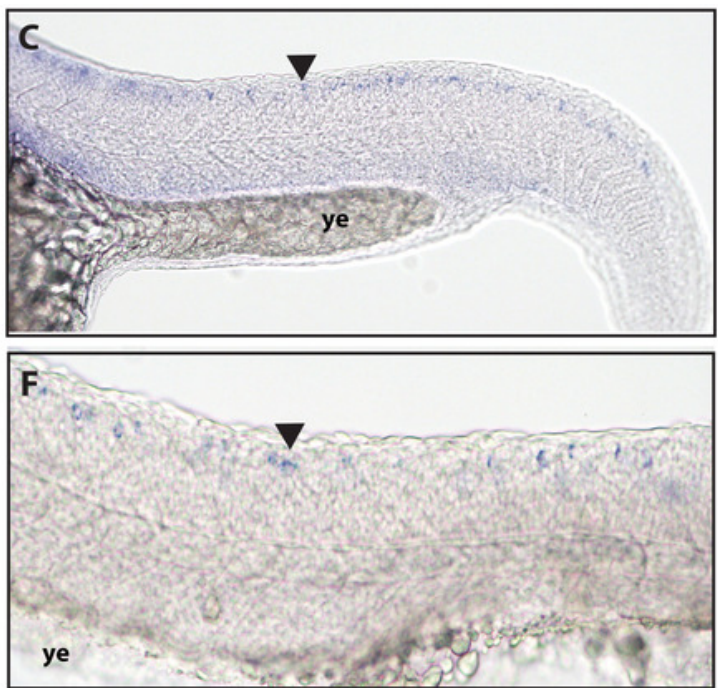


\section{Figure 2}

Early expression of $n t r k 2 a$ is seen in the head and spinal cord using whole mount in situ hybridization at $16.5 \mathrm{hpf}$ and $24 \mathrm{hpf}$.

Expression of $n$ trk2a is seen in the cranial ganglia (arrows) as well as in the RB neurons of the spinal cord (arrowheads) at (A \& D) $16.5 \mathrm{hpf}$ and $(B, C, E \& F) 24 \mathrm{hpf}$. The asterisk in $\mathrm{E}$ marks weak expression in the diencephalon that matches with previously published accounts. (A, B, C \& F) Lateral view. (D \& E) Dorsal view. All embryos are oriented with the head to the left. te, marks the telencephalon, mb, marks the midbrain, hb, marks the hindbrain, ov, marks the otic vesicle, chb, marks the caudal hindbrain and ye, marks the yolk extension. Data are representative of two rounds of in situ hybridization with at least 25 embryos per round.
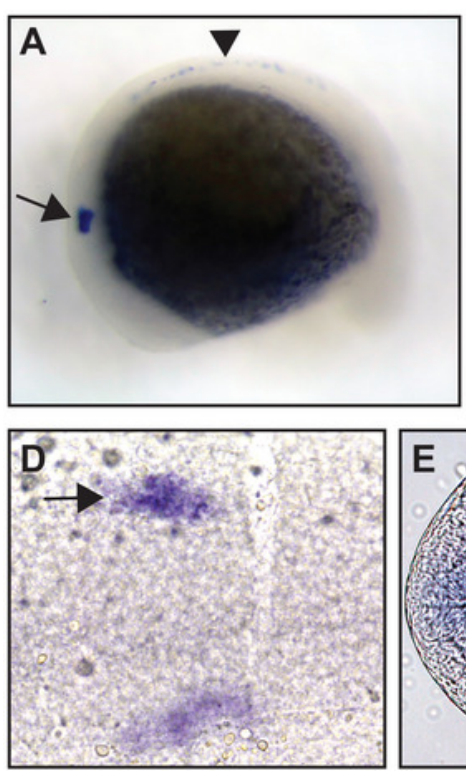
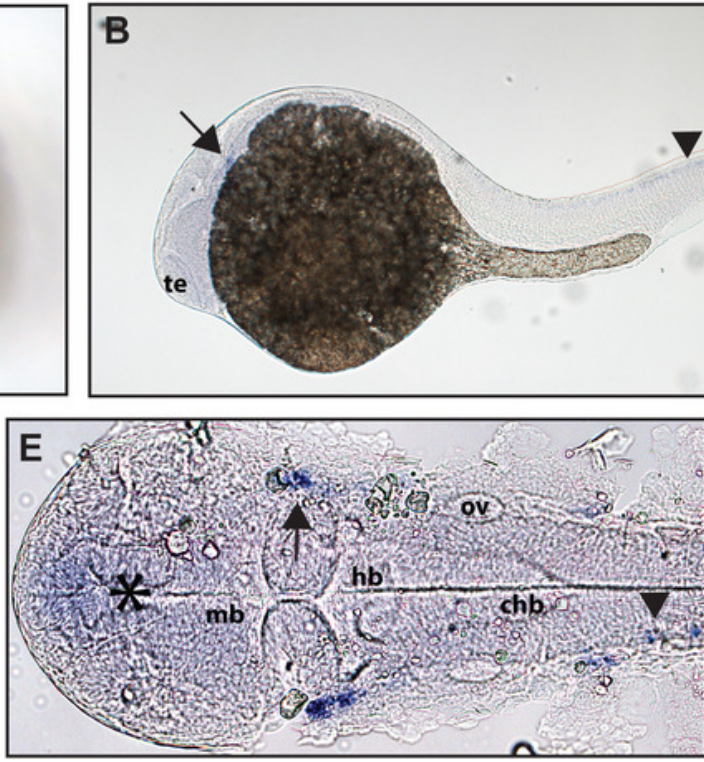
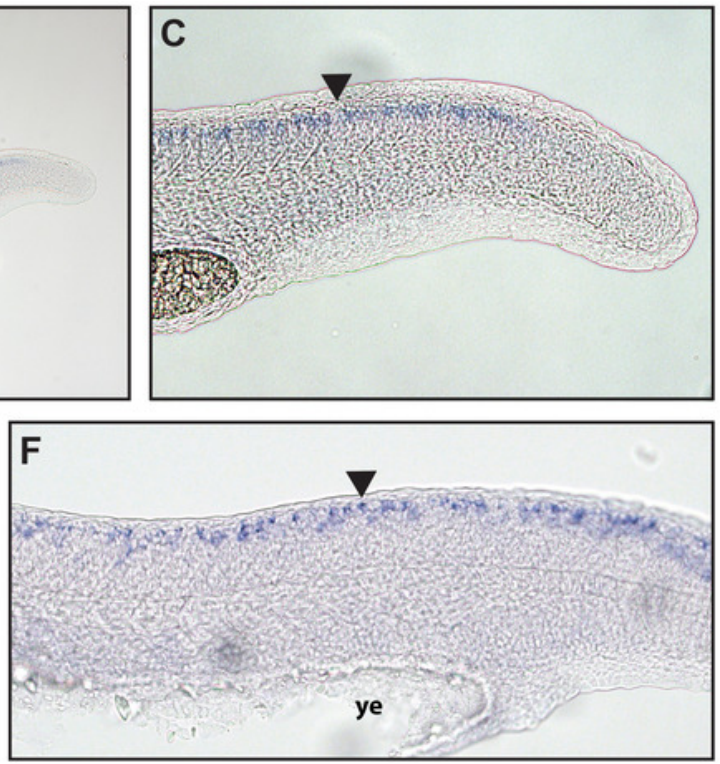


\section{Figure 3}

Early expression of p75 NTR ( $n g f r b$ ) is seen in two sets of cranial ganglia using whole mount in situ hybridization at $24 \mathrm{hpf}$.

$(A \& C)$ Expression of ngfrb was not detected at $16.5 \mathrm{hpf}$ and the staining that can be seen was non-specific (Supplemental Figure 2). (B, D \& E) At $24 \mathrm{hpf}$, expression of ngfrb was seen in two sets of cranial ganglia (arrows). (A, B \& E) Lateral view. ( $\&$ \&) Dorsal view. All embryos are oriented with the head to the left. $d$, marks the diencephalon, $c b$, marks the cerebellum, hb, marks the hindbrain, chb, marks the caudal hindbrain and ye, marks the yolk extension. Data are representative of the results from two rounds of in situ hybridization with at least 25 embryos per round.
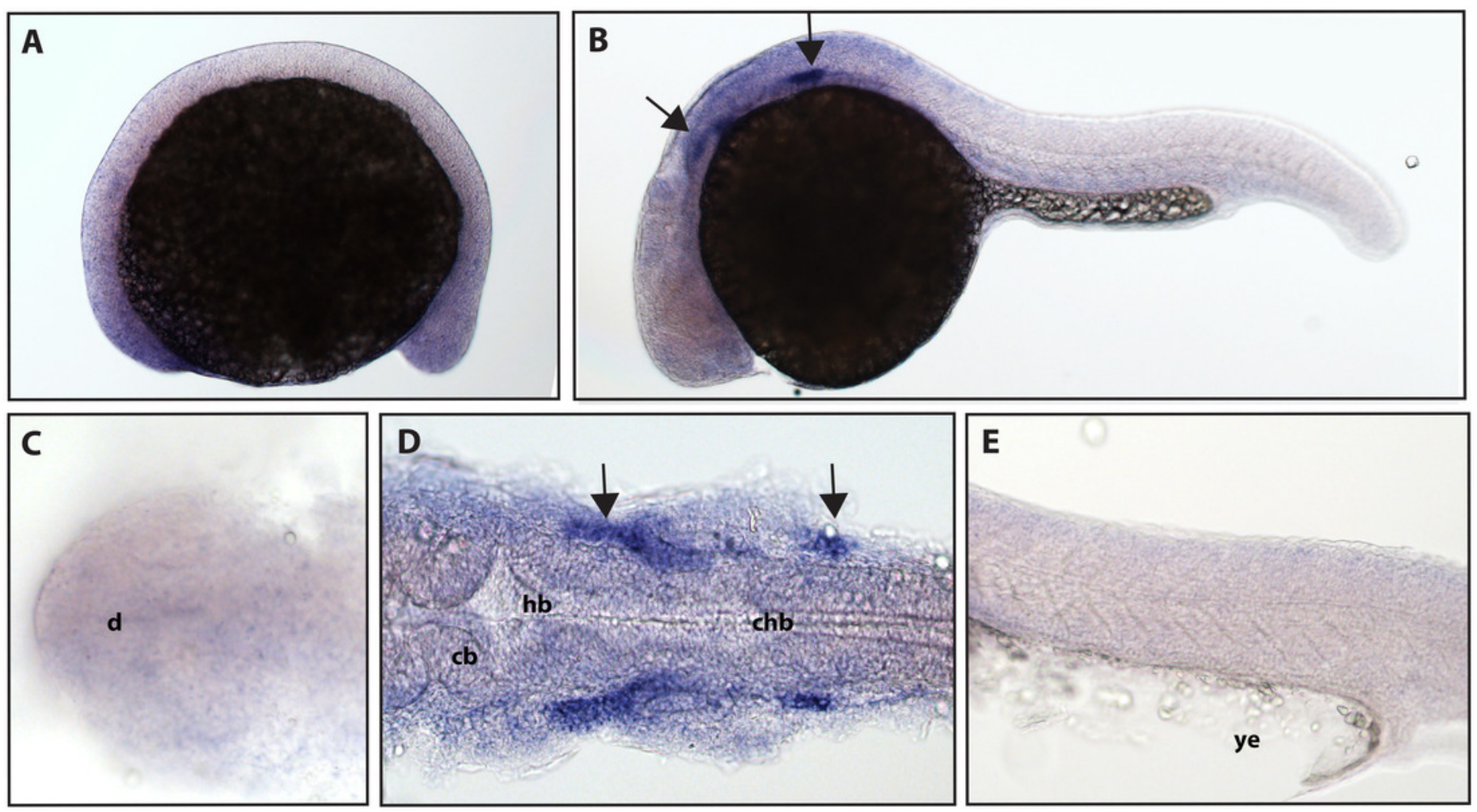


\section{Figure 4}

Early expression of $n t r k 2 b$ is seen in the head using whole-mount in situ hybridization at $24 \mathrm{hpf}$.

$(A \& C)$ Expression of $n t r k 2 b$ was not detectable at $16.5 \mathrm{hpf}$ (B, D \& E) At $24 \mathrm{hpf}$, expression of $n$ trk $2 b$ is seen in the telencephalon (arrows). ( $A, B \& E)$ Lateral view. ( $C \& D)$ Dorsal view. e, marks the eye, $d$, marks the diencephalon, mhb, marks the midbrain-hindbrain boundary, and ye, marks the yolk extension. Data are representative of three rounds of in situ hybridization with at least 25 embryos per round.
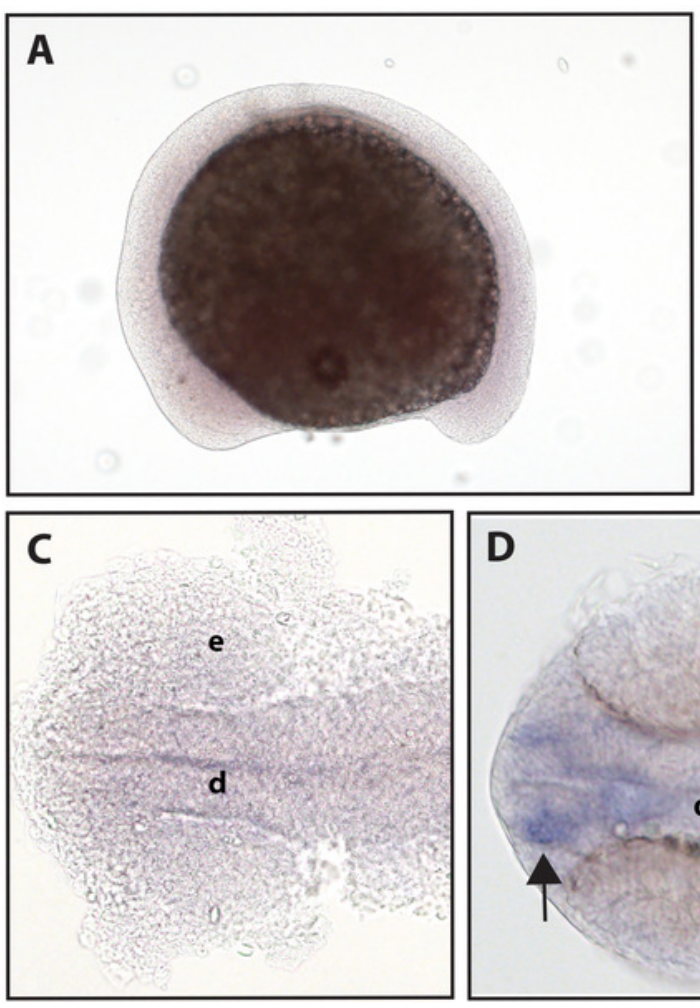
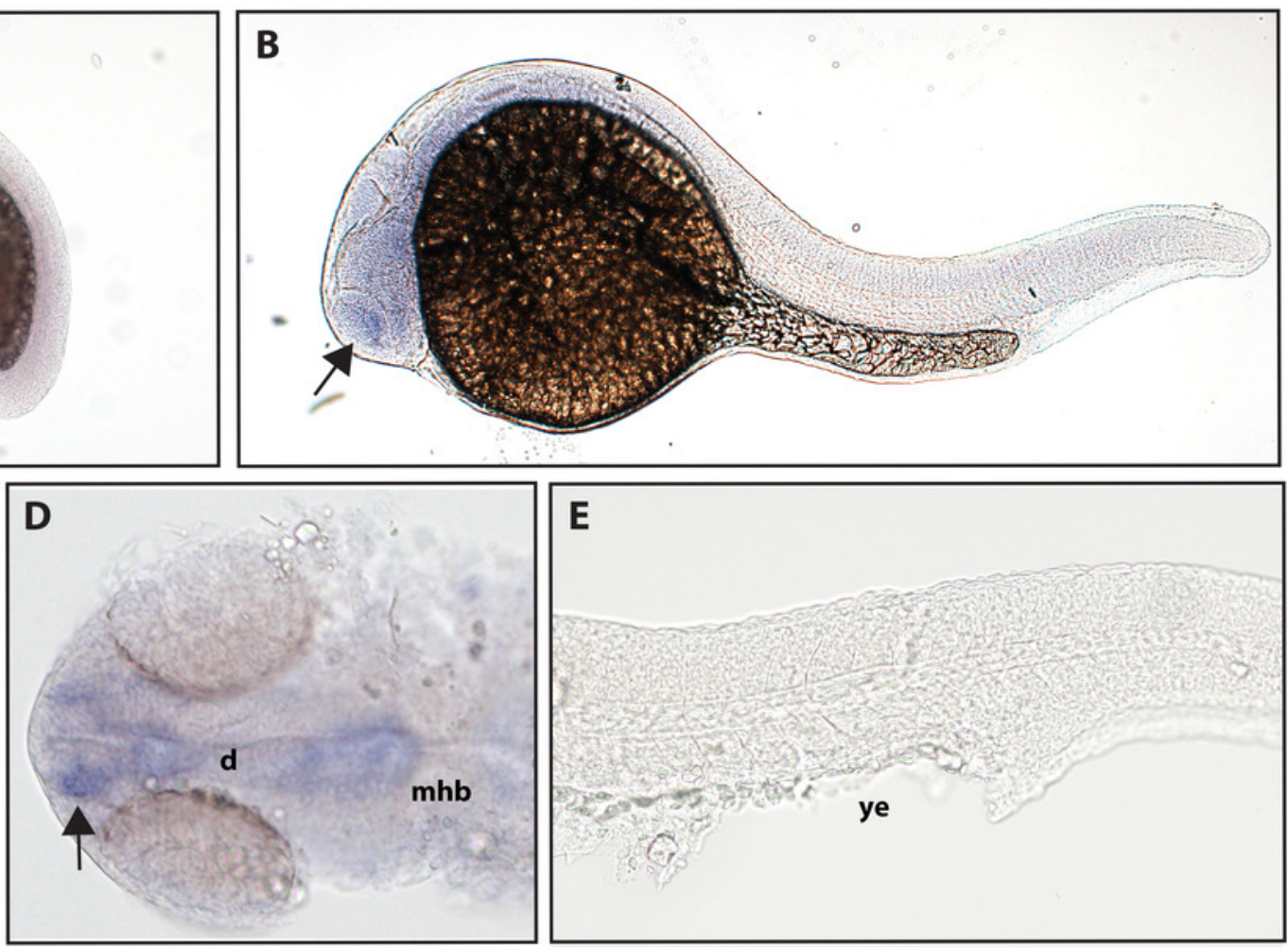

E 


\section{Figure 5}

Early expression of $n t r k 3 a$ is seen in the head and spinal cord using whole-mount in situ hybridization at $24 \mathrm{hpf}$.

$(A \& C)$ Expression of ntrk3a was not detected at $16.5 \mathrm{hpf}$ ( $B, D \& E)$ At $24 \mathrm{hpf}$, expression was seen in the telencephalon (arrow) and spinal cord (arrowheads). (A, B \& E) Lateral view. $(C \& D)$ Dorsal view. All embryos are oriented with the head to the left. e, marks the eye, $d$, marks the diencephalon, and ye, marks the yolk extension. This data is representative of the results from three rounds of in situ hybridization with at least 25 embryos per round.
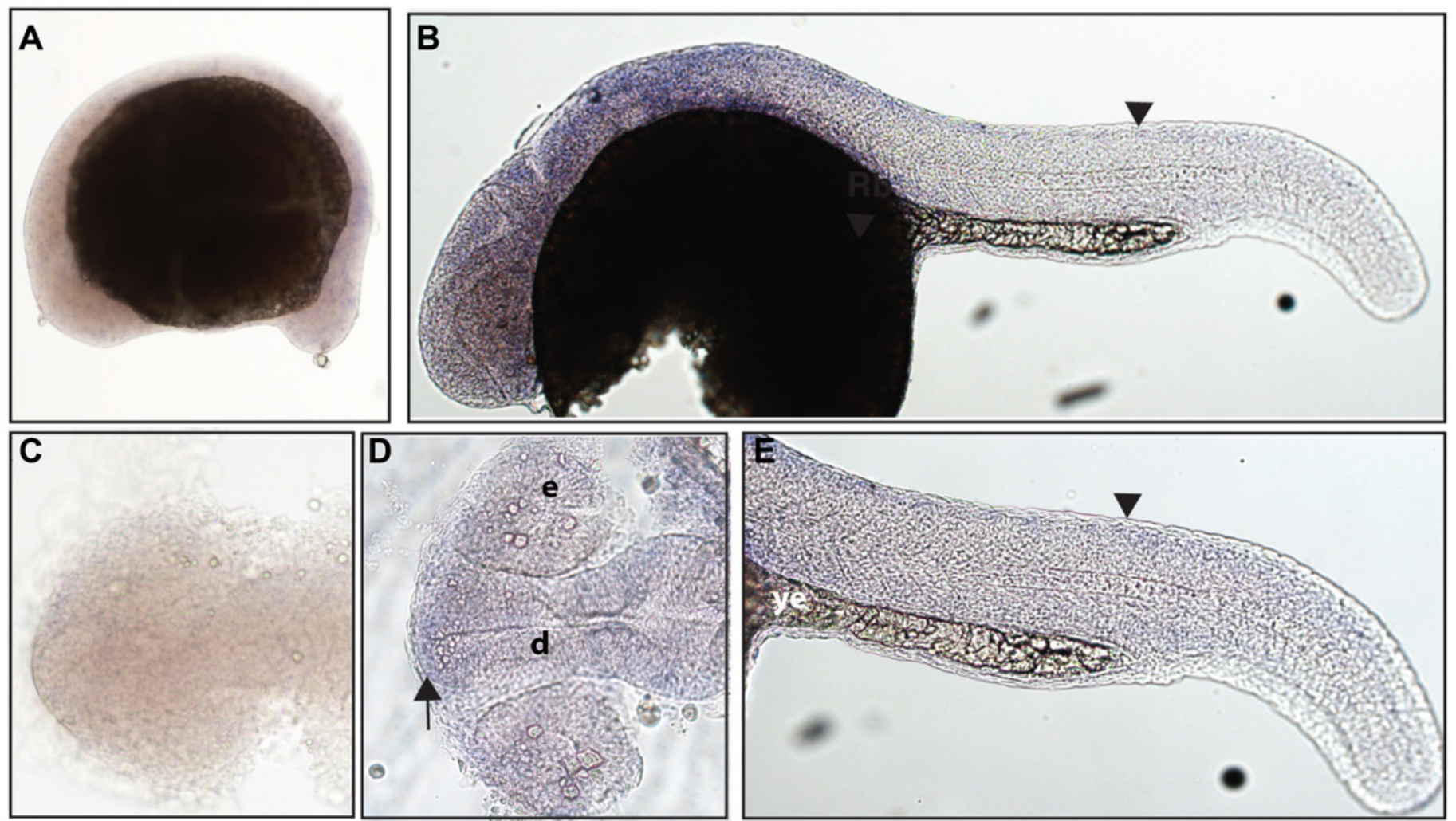


\section{Figure 6}

Early expression of $n t r k 3 b$ is seen throughout the head at $24 \mathrm{hpf}$ using whole mount in situ hybridization.

$(A \& C)$ At $16.5 \mathrm{hpf}$, no expression was detected. (B \& D) Expression was seen in the telencephalon (black arrows), the diencephalon (white arrows), the midbrain (red arrows), the hindbrain (blue arrows), and otic vesicle (black arrowhead). ( $\&$ \& $B$ ) Lateral view. (C \& D) Dorsal view. e, marks the eye, cb, marks the cerebellum, and chb, marks the caudal hindbrain. All embryos are oriented with the head to the left. This data is representative of the results of three rounds of in situ hybridization with at least 25 embryos per round.
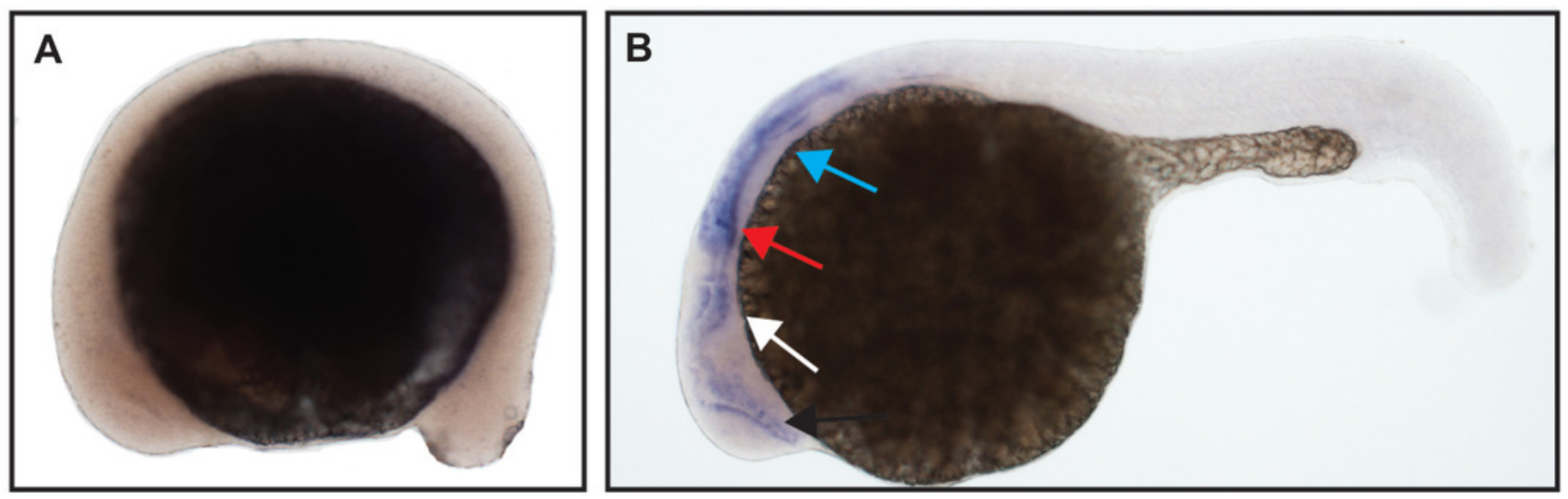

\section{C}

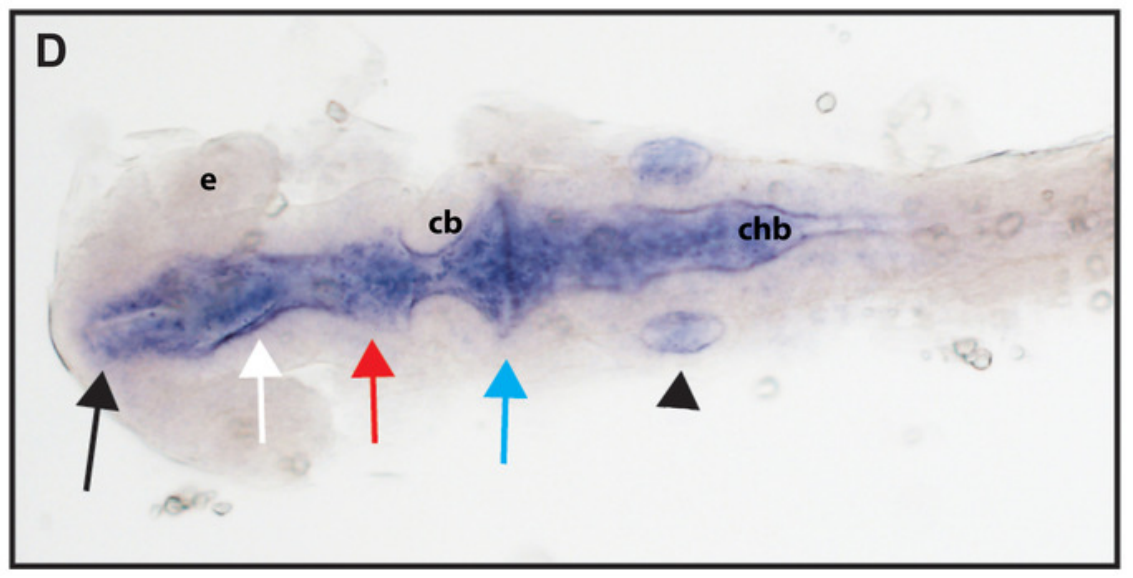

